\title{
Mixed Category Laboratory Procedure
}

National Cancer Institute

\section{Source}

National Cancer Institute. Mixed Category Laboratory Procedure. NCI Thesaurus. Code C74957.

A laboratory procedure that is performed in more than one of these laboratory specializations: anatomic pathology, clinical pathology, or molecular pathology. 